\title{
Next Generation Automated Software Evolution Refactoring at Scale
}

\author{
James Ivers \\ jivers@sei.cmu.edu \\ Software Engineering \\ Institute \\ Carnegie Mellon University \\ Pittsburgh, PA, USA
}

\author{
Ipek Ozkaya \\ ozkaya@sei.cmu.edu \\ Software Engineering \\ Institute \\ Carnegie Mellon University \\ Pittsburgh, PA, USA
}

\author{
Robert L. Nord \\ rn@sei.cmu.edu \\ Software Engineering \\ Institute \\ Carnegie Mellon University \\ Pittsburgh, PA, USA
}

\author{
Chris Seifried \\ seifried@sei.cmu.edu \\ Software Engineering \\ Institute \\ Carnegie Mellon University \\ Pittsburgh, PA, USA
}

\begin{abstract}
Despite progress in providing software engineers with tools that automate an increasing number of development tasks, complex activities like redesigning and reengineering existing software remain resource intensive or are supported by tools that are error prone. Complex, but common tasks in industry, like evolving large codebases $(1 \mathrm{M}+\mathrm{SLOC})$ to meet changing needs, still rely on costly manual efforts and incur significant technical risk. In one example, an organization that we work with estimated 14,000 hours of development work alone (excluding integration and testing) to isolate a feature from the underlying hardware platform. These examples are pervasive in industry. Software engineering research has taken providing effective tools for software evolution for granted for far too long. The time is right for research to take advantage of advances in search-based software engineering and create the next generation of industry-relevant automated software evolution tools. This paper lays out a vision for automated refactoring at scale towards this goal.
\end{abstract}

\section{CCS CONCEPTS}

- Software and its engineering $\rightarrow$ Software evolution; Software maintenance tools; Search-based software engineering; Software architectures.

\section{KEYWORDS}

refactoring at scale, search-based software engineering, automated software engineering, feature isolation

\section{ACM Reference Format:}

James Ivers, Ipek Ozkaya, Robert L. Nord, and Chris Seifried. 2020. Next Generation Automated Software Evolution Refactoring at Scale. In Proceedings of the 28th ACM foint European Software Engineering Conference and Symposium on the Foundations of Software Engineering (ESEC/FSE '20), November 8-13, 2020, Virtual Event, USA. ACM, New York, NY, USA, 4 pages. https://doi.org/10.1145/3368089.3417042

Permission to make digital or hard copies of all or part of this work for personal or classroom use is granted without fee provided that copies are not made or distributed for profit or commercial advantage and that copies bear this notice and the full citation on the first page. Copyrights for components of this work owned by others than ACM must be honored. Abstracting with credit is permitted. To copy otherwise, or republish, to post on servers or to redistribute to lists, requires prior specific permission and/or a fee. Request permissions from permissions@acm.org.

ESEC/FSE '20, November 8-13, 2020, Virtual Event, USA

(C) 2020 Association for Computing Machinery.

ACM ISBN 978-1-4503-7043-1/20/11.

https://doi.org/10.1145/3368089.3417042

\section{INTRODUCTION}

Successful systems endure, but they must contend with a steady stream of new requirements, technology changes, and new deployment targets. Almost inevitably, some kind of pervasive structural change is needed to meet these challenges. A simple, but common example today is the need to partition legacy monoliths into smaller pieces to create separately deployable, scalable, and evolvable microservices. These changes are costly, complicated, and risky, yet failing to implement them incurs significant consequences [9]. Most organizations that we work with are hobbled by different flavors of modernization challenges. The U.S. government alone spent over $\$ 90$ billion for system maintenance and operation in 2019 [15]. We know too well Lehman's law of continuing change [10]; software evolution is not a one-time problem for any system and will not cease to be a challenge for software engineers any time soon!

Experienced architects and developers have a common vocabulary to express the design direction they would like to take their software. However, going from this language of design to the programming language level changes required to realize their plans is a big leap. Research more commonly addresses the easier problems of assessing software or at best recommending quality-based improvements, mostly focusing on improving a metric with an unproven assumption that a cleaner codebase will ease evolution challenges. Consequently, developers must navigate the complex tasks of identifying what to change and how, manually executing the changes on all relevant development artifacts, and hoping for the best outcomes from substantive changes that affect dozens to hundreds of files.

We need to create tools that allow developers to express their design changes and trust that automation can correctly implement most of these changes in code. Examples include: disentangling the system around cross-cutting goals; standardizing data storage, replication, and access needs; and introducing fault management and security strategies. Industry-driven research to address complex engineering activities, particularly automated refactoring at scale to support system evolution, is long overdue. We posit that searchbased software engineering techniques, static code analysis tools, and the established design, architecture, and refactoring knowledge present an opportunity to create the next generation automated software evolution tools.

\section{RELATED WORK}

Murphy-Hill and Black [13] introduced a vocabulary for the need to continually tweak a codebase (floss refactoring) and infrequent, 
but focused changes to improve unhealthy code (root-canal refactoring). Their observations recognized that in order for software to be evolvable, refactoring operations should fit seamlessly within the implementation flow of developers, such as choosing a refactoring strategy quickly or switching between programming and refactoring. Floss and root-canal refactoring are differentiated by their frequency and health implications - the latter is harder, less common, more painful, and suggests prolonged quality neglect in a codebase. Tools that support either floss or root-canal refactoring treat software systems homogenously-refactoring to improve a metric or remove a code smell. However, this work doesn't necessarily provide consistent system-level restructuring.

We define refactoring at scale as restructuring software for a system-specific business goal, often around achieving a functional (reusing significant capabilities) or architectural change (migrating to microservices). The essence of these operations involves identifying relevant dependencies between software elements and resolving them against the target goal. This scope of refactoring pushes automation to solve specified problems instead of looking for easy wins and to generate solutions that should be assessed against business goals instead of general quality targets.

\subsection{Tools of the Trade}

Commercial and open source tools (e.g., SonarQube, Understand, Lattix, NDepend) identify structural couplings and generate warnings based on metrics and heuristic thresholds. However, these tools do not assess how changes can impact a specific objective, resulting in a high noise and false alarm rate. Modern development environments implement a subset of common refactorings like Rename, Extract Method or Extract Class, but, they do not offer recommendations. Moreover, they have yet to earn the trust of developers[4].

Fowler promoted the use of refactoring to improve the structure of software with a catalog of common refactorings that could be used to remove code smells [7]. The refactoring body of knowledge identifies a range of potential code changes, but leaves the selection of options and where to apply them to developer judgment. Current research in refactoring focuses on how refactoring improves code quality [5, 6], code smells [16], and how to select refactorings to do so [18]. However, most commercial and research advances don't take design trade-offs among competing objectives into account and may generate incorrect fixes, for example inserting a dependency where one should not be inserted.

\subsection{Why Now?}

Realizing refactoring at scale for hard evolution tasks requires a shift in framing the problem. Changes are pervasive (affecting lots of code), driven by evolution goals, and need to be assessed together rather than independently. The solutions are not deterministic, but involve balancing multiple trade-offs in context. Searchbased refactoring has been applied to frame refactoring selection as an optimization problem. Multi-objective algorithms provide an opportunity to incorporate design trade-offs into refactoring at scale. Current approaches incorporate heuristics to generate a set of Pareto optimal solutions $[1,8,11]$. Mkaouer et al. integrate quality attribute metrics to address design tradeoffs [12] and identify refactoring opportunities [2]. However, studies demonstrate little correlation between the opinions of developers and popular quality metrics, suggesting that these approaches may not produce useful results [19].

\section{AUTOMATING REFACTORING AT SCALE}

Our vision of refactoring at scale calls for automation that takes direction from developers in the language of design and automates the code changes required to realize those changes. This enables developers to shift work from tedious manual refactoring to confirming changes made by tools. Such next generation tools won't be easy to create, with many open questions of both research and pragmatic significance to address.

- How do we characterize project-specific goals? Users should provide their preferences as input. Automation should help users interactively refine or validate their specifications, such as through correlation with the results of feature location tools or application of static analysis and heuristics to explore the edges of a specification. We should strive to make tools tolerant to errors in preference expression.

- What portion(s) of a refactoring process should be automated? Automating tedious, repetitive, and error prone activities is a clear starting point. Crawling through thousands of dependencies is one such example. Should tools change the bare minimum to achieve a refactoring goal or should they look for opportunities to improve code along the way (e.g., by introducing design patterns)? Can we refactor database schema and stored procedures together with code? Tools should refactor test suites along with code. Can they also create unit tests as a byproduct of refactoring at scale?

- What solutions are acceptable to developers? Developers routinely navigate trade-offs among competing concerns like performance, security, and maintainability; tools need to do the same, but context matters. Research is needed to understand when criteria can simply be given weights, when developers need the option to accept/reject changes (and at what granularity), and when selecting a solution from a Pareto front of options is the best means of interaction.

- What representations enable successful refactoring at scale? Graph based representations allow dependencies to be captured and searched efficiently without all of the details of abstract syntax trees. Extensible representations allow data from different sources including static and dynamic analyses to be integrated, as well as incorporating information from code, data and other sources. Richer representations allow for refactorings that balance a greater number of concerns, such as refactoring software together with databases that they use.

- What balance of theoretical and pragmatic solutions do we aim for? Preserving behavior during refactoring is essential, but non-trivial, particularly with theoretical challenges like aliasing. Instead of striving for a general aliasing analysis, can we make progress with partial solutions that solve common, simpler problems? Tsantalis and Chatzigeorgiou, for example, define conditions under which a Move Method refactoring needn't consider aliasing to preserve semantics 
[17]. Tools should incorporate many such cases that enable reliable solutions without expensive or unsolvable analyses.

- How generalizable can automation be? Java, $\mathrm{C}++$, and $\mathrm{C \#}$ are all modern object-oriented programming languages, but their semantics differ in clear and subtle ways. How much of a refactoring can be defined against a common intermediate representation and how much must vary with programming language (e.g., accounting for the differences between explicit interfaces and multiple inheritance)?

- How should developer workflows change? Developers typically can only explore a few refactoring solutions. Tools, however, can quickly generate dozens of viable solutions and working code for each. How should new workflows distribute time among reviewing, testing, and benchmarking activities? How can tools ease the visualization of trade-offs to assist developers in decision making?

\subsection{What Is Feasible Today?}

In support of this vision, we are working on an automated design refactoring assistant to assist developers with feature isolation, one important problem in refactoring at scale. Accomplishing this kind of restructuring cost effectively is essential in evolving existing software to modern architectures and infrastructures. Feature isolation is the task of isolating the software that implements a feature from surrounding software [3]. Common industry scenarios that rely on feature isolation include:

- breaking monoliths into microservices to enable independent deployment, scaling, testing, evolution, etc.

- extracting features for reuse after system retirement, as a common asset across products, or rehosting on a new platform

- replacing a feature with an improved or less proprietary option

There are certainly techniques and technologies that assist in these scenarios, but none is a silver bullet. For example, domain driven design is one approach for finding seams along which to identify separable units, but what is easy to decouple may not align with features that have business value, and tool support is limited to model-based engineering tools [14].

Instead of looking across the whole code base for features that are easy to isolate, an effective tool works to solve a specific problem at a specific location in the code provided by a developer. We use a search-based software engineering approach that integrates multi-objective genetic algorithms, static code analysis, and the refactoring body of knowledge. Our approach includes:

Developing an approach to specify the feature that a developer would like to isolate: A project-specific problem (e.g. extract video surveillance feature) is specified as a list of user-defined types, such as classes, interfaces, etc., comprising the feature to isolate (our data range from a few dozen to a few hundred classes per feature). Source code is also an input (our examples scale up to 1.2M SLOC

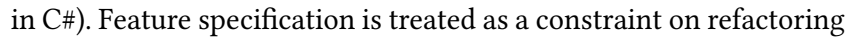
recommendations, i.e. every class in the specification must stay in the isolated feature, though other elements like classes, methods, interfaces can be added to the feature during refactoring.
Creating a viable representation: We use static code analysis to build an extensible graph of $\mathrm{C}$ \# constructs that are rich enough to reason about refactorings and that focus more on dependencies among types than on loop semantics within a method.

Creating a catalog of formalized refactorings: We derive refactoring operations from the refactoring literature and formalize each in terms of the graph (pre-conditions and graph transformations). This library of formalized refactorings allows algorithmic exploration of the solution space. Each is more constrained than Fowler's general refactorings, but constraints enable their formalization and assurance that each preserves behavior.

Developing a refactoring recommendation engine by applying search-based software engineering: Our goal is to recommend refactorings that resolve as many problematic couplings as possible against multiple objectives. Our library of fitness functions captures diverse developer concerns. Current examples include the degree of isolation (counts of problematic couplings), feature bloat (how much code is added to a feature), amount of work to be performed after refactoring such as code inspection, regenerating unit tests, or re-certification activities, and quality of the resulting code. We combine these elements through a multi-objective genetic search algorithm that explores different combinations of refactorings applied to different portions of the code to solve the project-specific feature isolation problem. Feature specification constrains the search process through a bias in mutation operations.

Developer reactions to early demonstrations show a hunger for automation to assist evolution. Recommended sequences of refactorings (Figure 1) were easy for developers to grasp and included sufficient information (where each refactoring was to be applied and with what parameters) to allow developers to review and validate the changes. Developers welcomed the accompanying data that differentiates refactorings that provide high value (solving big portions of the problem) from those that provide diminishing returns (perhaps indicating a point at which automation should suggest switching to human intervention as part of the solution). Developers also saw how the solution connected to their proposed scenarios. They reported that with such automated support they could get buy-in to do some of the big evolutionary tasks that had been in their backlog for years. Moreover, the ability to inspect what changes are recommended increases their confidence.

This kind of automation is certainly not a complete solution. Isolating the code that implements a feature is a necessary step, but not a sufficient one. Particularly in data heavy systems, the underlying database also needs to be refactored to make each microservice independent. Additional research is required to automate refactoring of databases for these scenarios and then to integrate the refactoring of code and data to provide solutions that explicitly manage the trade-offs between code and data changes.

A byproduct of this kind of approach can serve other purposes. For example, the fitness function we use to size the isolation problem and the accompanying data on how different portions of code were entangled was of great interest to cost estimation professionals, in particular since the data is centered around the actual region of code that will change. They posited uses including validating contractor proposals or performing portfolio analysis to prioritize modernization efforts by seeing where limited maintenance budgets might provide greater value. 


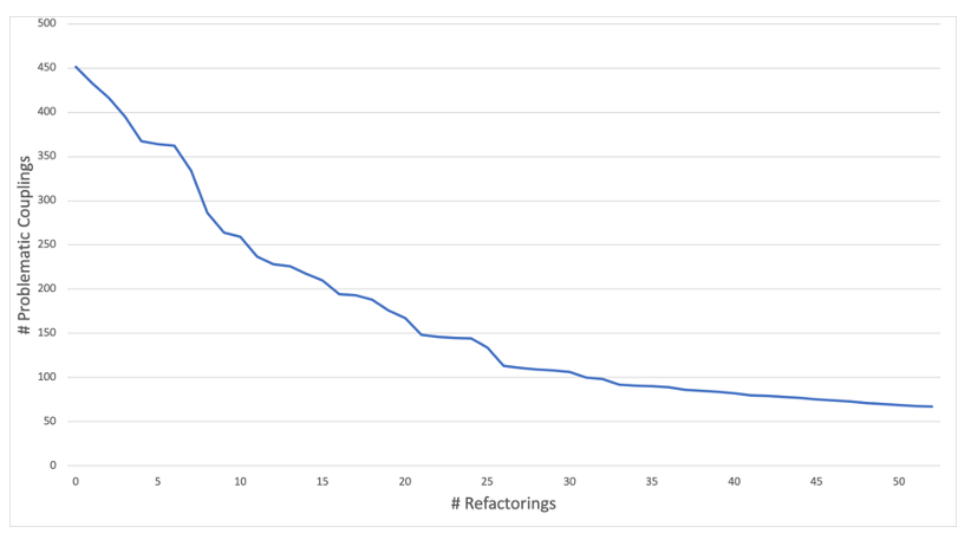

\author{
Best solution \\ Fitness $=67$ \\ Step 1: MoveClass (Duplicati.Server.WebServer.RESTMethods.RequestInfo) \\ Step 2: MoveClass (Duplicati.Server.Database.Backup) \\ Step 3: MoveClass (Duplicati.Server.Strings.Program) \\ Step 4: MoveInterface (Duplicati.Server.Serialization.Interface.ISetting) \\ Step 5: MoveClass (Duplicati.Library.Interface.CommandLineArgument) \\ Step 6: MoveClass (Duplicati.Server.LiveControls) \\ Step 7: MoveInterface (Duplicati.Server.Serialization.Interface.ISchedule) \\ Step 8: MoveInterface (Duplicati.Server.Serialization.Interface.IBackup) \\ Step 9: MoveInterface (Duplicati.Library.Interface.ICommandLineArgument) \\ Step 10: MoveClass (Duplicati.Server.Database.Schedule) \\ Step 11: MoveStaticMethod (Duplicati.Library.Localization.Short.LC.L \\ Step 12: ExtractClass (Duplicati.Library.Utility.Utility, \{ForceStreamRead, \\ ParseBoolOption, ClientFilenameStringComparison, ParseBool, \\ ReadFileWithDefaultEncoding, EPOCH $\}$ )
}

-...

Figure 1: Progress against the feature isolation goal (left) as recommended refactorings are added to a solution (right).

\section{CONCLUSION}

Continuing to strengthen the connection between software engineering research problems and industry business value can only improve relevance to industry problems and research impact. Automated solutions to assist refactoring at scale problems can reduce the cost of evolution tasks by orders of magnitude, resulting in many wins. Teams can overcome barriers like cost concerns by management or developers' fear of unintended consequences that have prevented the sought-after changes for years. Developers would focus on innovative tasks. Businesses would prolong their investments in valued features.

Search-based software engineering presents one class of solutions if oriented to solve the right problems. Next generation tools must solve user-specified problems, interact in the language of design, solve complex trade-off problems that span design and code, and generate solutions that developers will accept and use. The potential is incredible, as is the challenge of realizing that potential.

\section{ACKNOWLEDGMENTS}

This material is based upon work funded and supported by the Department of Defense under Contract No. FA8702-15-D-0002 with Carnegie Mellon University for the operation of the Software Engineering Institute, a federally funded research and development center. References herein to any specific commercial product, process, or service by trade name, trade mark, manufacturer, or otherwise, does not necessarily constitute or imply its endorsement, recommendation, or favoring by Carnegie Mellon University or its Software Engineering Institute. Document marking: DM20-0765.

\section{REFERENCES}

[1] M. Abebe and Cheol-Jung Yoo. 2014. Trends, Opportunities and Challenges of Software Refactoring: A Systematic Literature Review. Int. 7. Soft. Eng. Appl. 8, 6 (2014), 299-3183.

[2] Vahid Alizadeh, Mohamed Amine Ouali, Marouane Kessentini, and Meriem Chater. 2019. RefBot: Intelligent Software Refactoring Bot. In 34th IEEE/ACM Int. Conf. Conference on Automated Software Engineering, ASE 2019. IEEE, San Diego, CA, USA, November 11-15, 823-834. https://doi.org/10.1109/ASE.2019.00081

[3] Sven Apel and Christian Kästner. 2009. An Overview of Feature-Oriented Software Development. f. Object Technol. 8, 5 (2009), 49-84. https://doi.org/10.5381/ jot.2009.8.5.c5

[4] John Brant and Friedrich Steimann. 2015. Refactoring Tools are Trustworthy Enough and Trust Must be Earned. IEEE Softw. 32, 6 (2015), 80-83. https:
//doi.org/10.1109/MS.2015.145

[5] Mel Ó Cinnéide, Laurence Tratt, Mark Harman, Steve Counsell, and Iman Hemati Moghadam. 2012. Experimental assessment of software metrics using automated refactoring. In 2012 ACM-IEEE Int. Symp. on Empirpical Software Engineering and Measurement, ESEM '12. ACM, Lund, Sweden - September 19 - 20, 49-58. https://doi.org/10.1145/2372251.2372260

[6] Francesca Arcelli Fontana and Stefano Spinelli. 2011. Impact of refactoring on quality code evaluation. In Fourth Workshop on Refactoring Tools 2011, WRT '11, Danny Dig and Don S. Batory (Eds.). ACM, Waikiki, Honolulu, HI, USA, May 22, 37-40. https://doi.org/10.1145/1984732.1984741

[7] Martin Fowler. 1999. Refactoring Improving the Design of Existing Code. AddisonWesley, Boston, MA.

[8] Mark Harman and Laurence Tratt. 2007. Pareto optimal search based refactoring at the design level. In Genetic and Evolutionary Computation Conference, GECCO 2007, Proceedings. ACM, London, England, UK, July 7-11, 1106-1113. https: //doi.org/10.1145/1276958.1277176

[9] Philippe Kruchten, Robert Nord, and Ipek Ozkaya. 2019. Managing Technical Debt: Reducing Friction in Software Development. Pearson Addison-Wesley, Boston, MA.

[10] Meir M. Lehman. 1980. On understanding laws, evolution, and conservation in the large-program life cycle. F. Syst. Softw. 1 (1980), 213-221. https://doi.org/10. 1016/0164-1212(79)90022-0

[11] Thainá Mariani and Silvia Regina Vergilio. 2017. A systematic review on searchbased refactoring. Inf. Softw. Technol. 83 (2017), 14-34. https://doi.org/10.1016/j. infsof.2016.11.009

[12] Wiem Mkaouer, Marouane Kessentini, Adnan Shaout, Patrice Koligheu, Slim Bechikh, Kalyanmoy Deb, and Ali Ouni. 2015. Many-Objective Software Remodularization Using NSGA-III. ACM Trans. Softw. Eng. Methodol. 24, 3 (2015), 17:1-17:45. https://doi.org/10.1145/2729974

[13] Emerson R. Murphy-Hill and Andrew P. Black. 2008. Refactoring Tools: Fitness for Purpose. IEEE Softw. 25, 5 (2008), 38-44. https://doi.org/10.1109/MS.2008.123

[14] Florian Rademacher, Jonas Sorgalla, and Sabine Sachweh. 2018. Challenges of Domain-Driven Microservice Design: A Model-Driven Perspective. IEEE Softw. 35, 3 (2018), 36-43. https://doi.org/10.1109/MS.2018.2141028

[15] GAO Report. 2019. Agencies Need to Develop Modernization Plans for Critical Legacy Systems. GAO Report GAO-19-471. GAO.

[16] Nikolaos Tsantalis, Theodoros Chaikalis, and Alexander Chatzigeorgiou. 2018. Ten years of JDeodorant: Lessons learned from the hunt for smells. In 25th Int. Conf. on Software Analysis, Evolution and Reengineering, SANER 2018. IEEE Computer Society, Campobasso, Italy, March 20-23, 4-14. https://doi.org/10. 1109/SANER.2018.8330192

[17] Nikolaos Tsantalis and Alexander Chatzigeorgiou. 2009. Identification of Extract Method Refactoring Opportunities. In 13th European Conference on Software Maintenance and Reengineering, CSMR 2009, Architecture-Centric Maintenance of Large-Scale Software Systems. IEEE Computer Society, Kaiserslautern, Germany, 24-27 March, 119-128. https://doi.org/10.1109/CSMR.2009.23

[18] Wei Wang and Michael W. Godfrey. 2014. Recommending Clones for Refactoring Using Design, Context, and History. In 30th IEEE Int. Conf. on Software Maintenance and Evolution. IEEE Computer Society, Victoria, BC, Canada, September 29 - October 3, 331-340. https://doi.org/10.1109/ICSME.2014.55

[19] David R. White, Chris Simons, Jeremy Singer, and David White. 2015. Searchbased refactoring: Metrics are not enough. Lecture Notes in Computer Science 9275 (2015), 47-61. https://doi.org/10.1007/978-3-319-22183-0_4 